\title{
Primary Appendiceal Mucinous Adenocarcinoma
}

\author{
Prativa Kumari Behera • Pramod Kumar Rath • \\ Rabiratna Panda $\cdot$ Sanghamitra Satpathi • \\ Rajan Behera
}

Received: 8 January 2009 / Accepted: 18 January 2009/Published online: 3 December 2010

(C) Association of Surgeons of India 2010

\begin{abstract}
Primary Adenocarcinomas of the appendix are extremely rare tumor. We report a case of primary mucinous adenocarcinoma in a 40 year old lady misdiagnosed as having acute appendicitis. All the routine investigations were within normal limit. USG of abdomen showed dilated appendix with little fluid collection adjacent to it and no other abnormality was seen which suggested acute appendicitis. Appendicectomy was done and excised appendix was sent for histopathological examination. Mucinous Adenocarcinoma of the appendix was confirmed after histopathological examination. Right hemicolectomy was done as a second stage procedure. As some cases are incidentally discovered, this case emphasizes that histological examination of all appendicectomy specimens is mandatory.
\end{abstract}

Keywords Appendicitis · Biopsy $\cdot$ Appendix $\cdot$ Mucinous adenocarcinoma

\section{Introduction}

Primary appendiceal adenocarcinomas (PAAs) are very rare malignant neoplasm accounting for $0.05-0.2 \%$ of all appendectomies and only $6 \%$ of all malignant tumors of appendix [1]. It constitutes $<0.5 \%$ of all gastrointestinal neoplasm [2]. Recently PAAs have been classified into 4 groups: mucinous adenocarcinoma, colonic type adenocarcinoma, goblet cell carcinoma, signet ring cell carcinoma [3]. An exceptionally rare type of PAAs is signet ring type

P. K. Behera $(\bowtie) \cdot$ P. K. Rath $\cdot$ R. Panda $\cdot$ S. Satpathi $\cdot$ R. Behera Ispat General Hospital,

Rourkela, India

e-mail: pk_rkl@yahoo.in and most common is colonic type. Carcinomas of appendix are usually well differentiated mucinous adenocarcinoma, which tend to produce pseudomyxoma peritonei and do not show metastatic spread until late in the disease process. In contrast to adenocarcinoma of colon and rectum rarely result in pseudomyxoma peritonei and frequently metastasize even if mucinous and well differentiated [4]. We report a rare case of mucinous adenocarcinoma in a 40 years women misdiagnosed as having acute appendicitis.

\section{Case Report}

A 40 year old woman presented with acute abdominal pain of 3 days duration, pain was associated with vomiting and low grade fever. There was no history of loss of appetite, weight loss or any symptoms of bowel and bladder involvement, also no past history of similar attack. Systemic examination was within normal limits but abdominal examination revealed tenderness in right iliac fossa only. All routine investigations were within normal limits. Ultrasonography (USG) of the abdomen showed a dilated non compressible tubular lesion in the right iliac fossa with an adjacent hypo-echoic lesion (Fig. 1). The possibility of the lesion being an inflamed appendix was suggested. Appendectomy was done. An intra-operative diagnosis of acute appendicitis was made, as the appendix was dilated, inflamed and there was little fluid collected around it. No mucinous material was found inside the abdominal cavity. This specimen was sent for histopathological examination. On gross examination the appendix was enlarged to $10 \mathrm{~cm} \times 4 \mathrm{~cm}$ in size, soft in consistency and reddish in color. Cut section showed dilated lumen filled with small amount of mucus. Histopathology confirmed mucinous adenocarcinoma of appendix as there was 
Fig. 1 USG showing dilated appendix with fluid collection adjacent to it

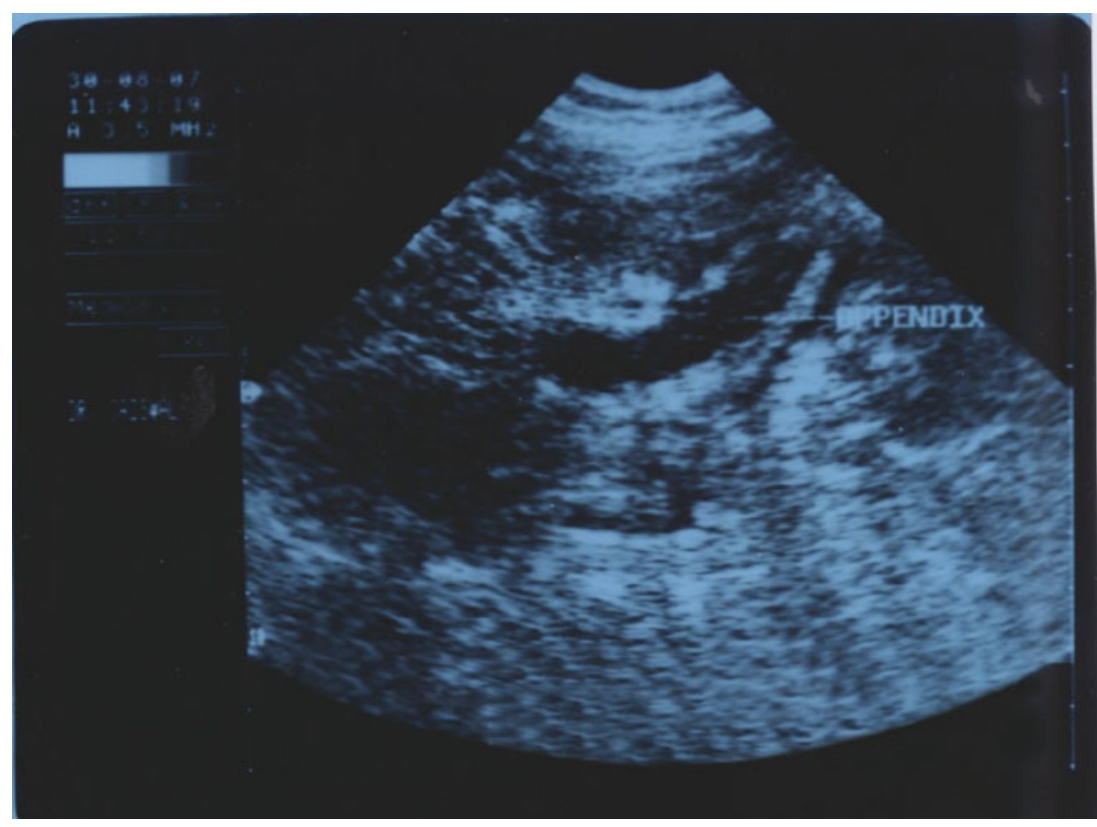

invasion of the appendiceal wall by malignant cells forming glands and extra cellular mucin (Fig. 2). So right hemicolectomy was done as a second stage procedure.

\section{Discussion}

Adenocarcinoma of the appendix is extremely rare. In our institute in over last 7 years (2002-2008)700 appendicectomy specimens were analyzed. This was the only case of primary mucinous adenocarcinoma of appendix. In 15 to $20 \%$ of cases a second malignancy in the gastrointestinal tract is associated with the disease [5]. Adenocarcinoma of appendix most often present as acute appendicitis or as a palpable abdominal mass. But some are entirely asymptomatic. Malignancy of the appendix are never suspected preoperatively and seldom intra

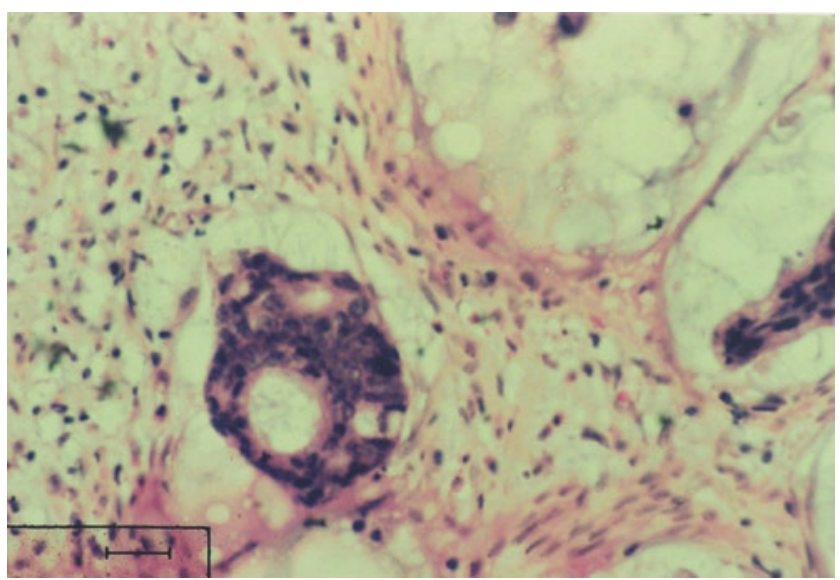

Fig. 2 Photomicrograph showing invasion of the appendiceal wall by malignant cells forming glands and extra cellular mucin $(H \& E, \times 40)$ operatively, the diagnosis being usually made at histopathological examination of the surgical specimen or as an incidental finding during exploration for another disease [3]. The mean age at presentation for mucinous type PAA is about 50 years with a male pre dominance of 4:1 [6]. Most mucinous type PAAs are well differentiated slowly growing pushing rather than infiltrating margin. Its importance lies in the treatment consideration and tendency to produce condition such as pseudomyxoma peritonei. The presence of mucin within the peritoneal cavity has been classically referred to as pseudomyxoma peritonei. But the designation should be reserved for the cases in which the condition is wide spread rather than limited to a small pull of mucin around the appendix and in which epithelial tumor cell are identifiable microscopically [7]. From treatment and prognostic stand point the distinction between benign mucinous conditions and mucinous adenocarcinoma of appendix is of great importance. At histopathology, invasion of the appendiceal wall by the atypical glands and identification of epithelial cell in any intra peritoneal mucinous collection, if present are the feature of malignancy [8]. Mucinous histology has a better prognosis than the colonic histology. Mucinous and non mucinous carcinoma of the appendix has similar genetic alteration but different clinical presentation and prognosis [9]. The traditional treatment for mucinous adenocarcinoma confined to the appendix is Right hemicolectomy [10]. But in case of intra abdominal metastasis the treatment consists of aggressive debulking followed by adjuvant chemoradiotherapy along with it [11]. Since distant metastasis and visceral involvement are very rare, death occurs mostly due to loss of intestinal function and obstruction by peritoneal implant confined to the abdomen. The prognosis of mucinous adenocarcinoma depends 
on the grade of malignancy of the mucinous tumor and success of debulking surgery to remove all the tumors that have metastasized into the abdomen.

The case is very interesting because pre and intra operative diagnosis was acute appendicitis as the age was 40 ;sex was female and no feature of malignancy but histopathology confirmed it to be adenocarcinoma of appendix. Therefore histopathological examination of all appendicectomy specimens is mandatory to rule out malignant pathology. We are reporting this case because of rarity and also interesting. The incidence of PAA in our institute was $0.14 \%$ which correlates with that described in the literature.

\section{References}

1. Me O' Donnell, Badger SA, Beattie GC, Carson J, Garstin WI (2007) Malignant neoplasms of the appendix. Int J Colorectal Dis 22:1239-1248

2. Ozakyol AH, Saricom T, Kabukceaglu S, Caga T, Erenoglu E (1999) Primary appendiceal adenocarcinoma. Am J Clin Oncol 22(5):458459
3. Mc Cusker ME, Coti TR, Clag LX, Sobin LH (2002) Primary malignant neoplasm of the appendix. Cancer 94(12):3307-3312

4. Carr NJ, Emery TS, Sobin LH (2002) Epithelial neoplasm of the appendix and colorectum. Arch Pathol Lab Med 126(7):837-841

5. Lee J, Gardiner KR, Wilson BG (1996) Incidental mucinous cystadenocarcinoma of the appendix. Postgrad Med J 72(843):5556

6. Conte CC, Petrelli NJ, Stule J, Herrera L, Mittelman A (1988) Adenocarcinoma of the appendix. Surg Gynecol Obstet 166:451453

7. Smith JW, Kemeny N, Caldwell C, Banner P, Sigerdson E, Huro SA (1992) Pseudomyxoma peritonei of appendiceal origin. The memorial sloan Kettering Cancer centre experience. Cancer 70:396-401

8. Rutledge RH, Alexander JW (1992) Primary appendiceal malignancies: rare but important. Surgery 111:244-250

9. Kabbani W, Houlihan PS, Luthra R, Hamilton SR, Rashid A (2002) mucinous and non mucinous appendiceal adenocarcinoma, different clinic pathological features and similar genetic alteration. Mod Path 15(6):599-605

10. Lo NS, Sarr MG (2003) Mucinous cystadenocarcinoma of the appendix. The controversy persists: a review. Hepatogastroenterology 50(50):432-437

11. Topkan E, Polat Y, Karaoglu A (2008) Primary mucinous adenocarcinoma with chemotherapy and radiotherapy. A case report. Tumori 94:596-599 